\title{
15 PROGRAMAS DE INTERVENÇÃO PSICOTERAPÊUTICA GRUPAL: IMPLEMENTAÇÃO E AVALIAÇÃO NUM CONTEXTO DA PRÁTICA CLÍNICA
}

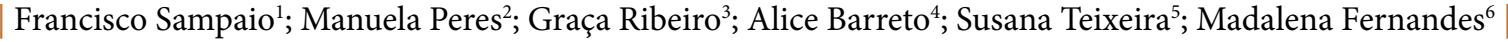

\section{RESUMO}

CONTEXTO: O Regulamento n. ${ }^{\circ}$ 129/2011 aponta os enfermeiros especialistas em Enfermagem de Saúde Mental como tendo competência para prestar cuidados de âmbito psicoterapêutico, socioterapêutico, psicossocial e psicoeducacional. Contudo, nos contextos da prática clínica em Portugal, apesar de se acreditar que são realizadas intervenções psicoterapêuticas por parte dos mesmos é escassa a divulgação dessas práticas.

OBJETIVO: Apresentar um percurso de implementação e avaliação de programas de intervenção psicoterapêutica grupal realizados num contexto da prática clínica.

MÉTODOS: Artigo de divulgação de boas práticas. Foram selecionados intencionalmente 153 participantes, divididos por programas de intervenção psicoterapêutica grupal dirigidos aos focos de Enfermagem "Autoestima", "Ansiedade" e "Sono", no período compreendido entre 23 de março e 31 de dezembro de 2015.

RESULTADOS: Todos os programas de intervenção são compostos por um conjunto de cinco sessões com duração entre 50 e 60 minutos. É realizada uma avaliação a priori e a posteriori dos focos alvo da intervenção com recurso a instrumentos psicométricos. Os programas de intervenção dirigidos aos focos "Ansiedade" e "Sono" revelaram a existência de diferenças estatisticamente significativas entre os scores inicial e final.

CONCLUSÕES: Parecem existir benefícios, para os utentes, da realização de programas de intervenção psicoterapêutica grupal dinamizados por enfermeiros especialistas em Enfermagem de Saúde Mental. Estes são dados que podem ser utilizados para argumentar junto dos gestores hospitalares e dos decisores políticos acerca do potencial e capacidade destes mesmos enfermeiros especialistas para executar intervenções deste género.

PALAVRAS-CHAVE: Enfermagem psiquiátrica; Psicoterapia; Unidade hospitalar de psiquiatria

\section{RESUMEN}

\section{"Programas de intervención psicoterapéutica grupal: Imple-} mentación y evaluación en un contexto de la practica clínica”

CONTEXTO: El Reglamento no 129/2011 señala los enfermeros especialistas en Salud Mental como competentes para proporcionar asistencia psicoterapéutica, socioterapéutica, psicosocial y psicoeducativa. Sin embargo, en el contexto de la práctica clínica en Portugal, aunque se considera que se llevan a cabo intervenciones psicoterapéuticas por los mismos, hay poca divulgación de este tipo de prácticas.

OBJETIVO: Para presentar una trayectoria de implementación y evaluación de programas de intervención psicoterapéutica grupal llevados a cabo en uno contexto de la práctica clínica.

MÉTODOS: Artículo de divulgación de buenas prácticas. Fueron seleccionados intencionalmente 153 participantes, divididos por los programas de intervención psicoterapéutica grupal dirigidos a los focos de atención de enfermería "Autoestima", "Ansiedad" y "Sueño", en el período comprendido entre el 23 de marzo y el 31 de diciembre de 2015.

RESULTADOS: Todos los programas de intervención se componen de un conjunto de cinco sesiones que duran entre 50 y 60 minutos. Se lleva a cabo una evaluación a priori y a posteriori de los focos albo de intervención con recurso al uso de instrumentos psicométricos. Los programas de intervención dirigidos a los focos "Ansiedad" y "Sueño" revelaron la existencia de diferencias estadísticamente significativas entre las puntuaciones inicial y final.

CONCLUSIONES: La realización de programas de intervención psicoterapéutica grupal conducidos por enfermeros especialistas en Salud Mental parece ser beneficiosa para los pacientes. Estos son datos que pueden ser utilizados para discutir con los administradores hospitalarios y con los responsables políticos acerca del potencial y de la capacidad de estos enfermeros especialistas para llevar a cabo intervenciones de este tipo.

DESCRIPTORES: Enfermería psiquiátrica; Psicoterapia; Servicio de Psiquiatría en Hospital

\section{ABSTRACT \\ "Group psychotherapeutic intervention programmes: Imple- mentation and evaluation in a clinical practice context"}

BACKGROUND: Regulation No 129/2011 states that providing psychotherapeutic, sociotherapeutic, psychosocial and psychoeducational care is a competence of mental health nurses. However, in the clinical practice in Portugal, although we believe that mental health nurses perform psychotherapeutic interventions, these are little publicised.

AIM: To present an implementation and evaluation path of group psychotherapeutic intervention programmes carried out in the clinical practice.

METHODS: Article of dissemination of good practices. One hundred and fifty-three (153) participants were intentionally selected and they were distributed into different group psychotherapeutic intervention programmes, which intended to address the nursing focuses "Self-Esteem", "Anxiety" and "Sleep", between 23rd March and 31st December 2015.

RESULTS: All the intervention programmes consist of a set of five sessions lasting between 50 and 60 minutes. An a priori and a posteriori evaluation of the target focus of the intervention was carried out by using psychometric instruments. The intervention programmes that addressed the focuses "Anxiety" and "Sleep" revealed the existence of statistically significant differences between the initial and the final scores.

CONCLUSIONS: Patients seem to benefit of group psychotherapeutic intervention programmes performed by mental health nurses. These data can be used to argue with decision makers and policy makers about the potential and capability of mental health nurses to perform interventions of this kind.

\section{KEYWORDS: Psychiatric nursing; Psychotherapy; Psychiatric department, hospital}

Submetido em 31-01-2017

Aceite em 30-04-2017

\footnotetext{
1 Mestre em Enfermagem de Saúde Mental e Psiquiatria; Doutorando em Ciências de Enfermagem no ICBAS; Assistente Convidado na ESEP; Investigador no Center for Health Technology and Services Research, Enfermeiro especialista em Enfermagem de Saúde Mental e Psiquiátrica no Hospital de Braga, francisco.sampaio@hospitaldebraga.pt 2 Licenciada em Enfermagem; Enfermeira especialista em Enfermagem de Saúde Mental e Psiquiátrica no Hospital de Braga, maria.manuela.lopes@hospitaldebraga.pt 3 Licenciada em Enfermagem; Enfermeira especialista em Enfermagem de Saúde Mental e Psiquiátrica no Hospital de Braga, Portugal, graca.ribeiro@hospitaldebraga.pt 4 Licenciada em Enfermagem; Enfermeira especialista em Enfermagem Médico-Cirúrgica no Hospital de Braga, Portugal, maria.barreto@hospitaldebraga.pt 5 Licenciada em Enfermagem; Enfermeira especialista em Enfermagem de Saúde Mental e Psiquiátrica no Hospital de Braga, Portugal, susana.teixeira@hospitaldebraga.pt 6 Licenciada em Enfermagem; Pós-Licenciatura de Especialização em Enfermagem Médico-Cirúrgica; Enfermeira-Chefe no Hospital de Braga - Serviço de Psiquiatria, 4710243 Braga, Portugal, maria.madalena.silva@hospitaldebraga.pt
}

Citação: Sampaio, F., Peres, M., Graça, R., Barreto, A., Teixeira, S., \& Fernandes, M. (2017). Programas de intervenção psicoterapêutica grupal: implementação e avaliação num contexto da prática clínica. Revista Portuguesa de Enfermagem de Saúde Mental (Spe. 5), 87-92. 


\section{INTRODUÇÃO}

A intervenção psicoterapêutica pode ser definida como uma atividade que envolve contactos sistemáticos e limitados no tempo entre uma pessoa em distress e alguém que tenta reduzir esse mesmo distress produzindo mudanças nos sentimentos, atitudes e comportamento da pessoa que apresenta sofrimento, por via de uma relação terapêutica (Frank in Bloch, 2006; Frank \& Frank, 1993). A definição apresentada parece apontar para a possibilidade de profissionais de saúde de áreas disciplinares diferentes realizarem intervenções psicoterapêuticas em contexto clínico, ideia essa que é corroborada pelo Regulamento n. ${ }^{\circ}$ 129/2011 (p. 8672) que, pese embora a sua ausência de valor legal, aponta os enfermeiros especialistas em Enfermagem de Saúde Mental como tendo competência para prestar "cuidados de âmbito psicoterapêutico, socioterapêutico, psicossocial e psicoeducacional".

O Regulamento n. ${ }^{\circ} 356 / 2015$, relativo aos Padrões de Qualidade dos Cuidados Especializados em Enfermagem de Saúde Mental, refere que a realização de intervenções psicoterapêuticas por parte dos enfermeiros especialistas em Enfermagem de Saúde Mental deve assentar, naturalmente, no estabelecimento de uma relação psicoterapêutica, caraterizando-se esta pela "evolução técnica e processual da ligação entre duas pessoalidades (o cliente individual ou grupal e a pessoa do enfermeiro) e uma profissionalidade (o enfermeiro e a profissão de enfermagem)" (p. 17040). Ainda assim, não é clara a tipologia de intervenções psicoterapêuticas que pode ser realizada pelos enfermeiros especialistas em Enfermagem de Saúde Mental sendo que, para um melhor entendimento deste aspeto, é necessário o recurso à literatura de referência no que concerne às intervenções de Enfermagem (Bulechek, Butcher, \& Dochterman, 2010), que aponta exemplos de intervenções psicoterapêuticas que se enquadram no domínio disciplinar e profissional de Enfermagem, tais como a reestruturação cognitiva (4700), o treino de controlo de impulsos (4370), e o aconselhamento (5240).

Ainda que existam dúvidas regulamentares, decorrentes da pouca clareza dos documentos oficiais elaborados relativamente a esta matéria, é absolutamente claro o reconhecimento da competência dos enfermeiros especialistas em Enfermagem de Saúde Mental, que poderia ser reforçada pela criação de um Modelo de Intervenção Psicoterapêutica em Enfermagem, sendo que já existe investigação a ser empreendida nesse sentido (Sampaio, Sequeira, \& Lluch Canut, 2015; Sampaio, Sequeira, \& Lluch Canut, 2016; Sampaio, Sequeira, \& Lluch Canut, 2017). Contudo, nos contextos da prática clínica, e especificamente em Portugal, apesar de se acreditar que são realizadas intervenções psicoterapêuticas, de forma estruturada, por parte dos enfermeiros especialistas em Enfermagem de Saúde Mental, é escassa a divulgação dessas práticas junto da comunidade científica.

Tendo em conta os dados anteriormente apresentados, e visando divulgar uma boa prática de Enfermagem realizada num serviço de saúde em Portugal, o presente artigo científico tem por objetivo apresentar um percurso de implementação e avaliação de programas de intervenção psicoterapêutica grupal realizados num contexto da prática clínica (Serviço de Internamento de Agudos de Psiquiatria de um Hospital da Região Norte de Portugal).

\section{Programas de Intervenção Psicoterapêutica Grupal}

No Quadro 1 são apresentadas as principais caraterísticas dos programas de intervenção psicoterapêutica grupal desenvolvidos para dar resposta aos focos de Enfermagem "Ansiedade", "Sono" e "Autoestima".

Quadro 1 - Caraterísticas dos Programas de Intervenção Psicoterapêutica Grupal

\begin{tabular}{|c|c|c|c|}
\hline Foco & Ansiedade & Sono & Autoestima \\
\hline $\begin{array}{l}\text { Número de } \\
\text { Sessões }\end{array}$ & \multicolumn{3}{|c|}{5} \\
\hline $\begin{array}{c}\text { Duração das } \\
\text { Sessões }\end{array}$ & \multicolumn{3}{|c|}{50 a 60 minutos } \\
\hline $\begin{array}{l}\text { Procedimen- } \\
\text { to Genérico }\end{array}$ & \multicolumn{3}{|c|}{$\begin{array}{c}\text { Sessões diárias, de } 2^{\mathrm{a}} \text { a } 6^{\mathrm{a}} \text { feira, no período } \\
\text { da manhã, em cada uma das alas (feminina e } \\
\text { masculina) do Serviço }\end{array}$} \\
\hline $\begin{array}{l}\text { Avaliação da } \\
\text { Intervenção }\end{array}$ & $\begin{array}{l}\text { A priori e a } \\
\text { posteriori } \\
\text { com recurso } \\
\text { à Escala de } \\
\text { Avaliação da } \\
\text { Ansiedade } \\
\text { de Hamilton }\end{array}$ & $\begin{array}{l}\text { A priori e a } \\
\text { posteriori com } \\
\text { recurso ao Ín- } \\
\text { dice da Quali- } \\
\text { dade do Sono de } \\
\text { Pittsburgh }\end{array}$ & $\begin{array}{l}\text { A priori e a } \\
\text { posteriori } \\
\text { com recurso } \\
\text { à Escala de } \\
\text { Autoestima } \\
\text { de Rosen- } \\
\text { berg }\end{array}$ \\
\hline $\begin{array}{l}\text { Conteúdo da } \\
\text { Intervenção }\end{array}$ & $\begin{array}{l}\text { Intervenção } \\
\text { baseada nos } \\
\text { princípios } \\
\text { teóricos do } \\
\text { treino de } \\
\text { resolução de } \\
\text { problemas }\end{array}$ & $\begin{array}{l}\text { Uma sessão de } \\
\text { Educação para a } \\
\text { Saúde + quatro } \\
\text { sessões de re- } \\
\text { laxamento (tre- } \\
\text { ino de relaxa- } \\
\text { mento muscular } \\
\text { e relaxamento } \\
\text { por imaginação } \\
\text { guiada }\end{array}$ & $\begin{array}{l}\text { Intervenção } \\
\text { baseada nos } \\
\text { princípios } \\
\text { teóricos do } \\
\text { treino de } \\
\text { resolução } \\
\text { de prob- } \\
\text { lemas }\end{array}$ \\
\hline
\end{tabular}


Os grupos de intervenção são fechados e compostos por um máximo de oito participantes que devem apresentar um problema comum (diagnóstico de Enfermagem previamente identificado, relacionado com um dos focos para os quais foram elaborados programas de intervenção). As sessões de intervenção psicoterapêutica grupal são executadas por um enfermeiro especialista em Enfermagem de Saúde Mental e Psiquiátrica que, nos dias úteis, tem como plano de trabalho único para o turno da manhã a realização das mesmas.

\section{Implementação dos Programas de Intervenção Psi- coterapêutica Grupal}

Sob o ponto de vista dos procedimentos adotados, numa primeira fase foi elaborado um projeto (aprovado pela Direção de Enfermagem da organização hospitalar) que tinha em vista a implementação de programas de intervenção terapêutica e psicoterapêutica grupal num Serviço de Internamento de Psiquiatria de um Hospital da Região Norte de Portugal, sendo que o presente artigo apenas se irá debruçar sobre os programas de intervenção psicoterapêutica grupal. Assim, numa fase inicial, e por se considerar que esses seriam os focos de Enfermagem mais relevantes no contexto da prática clínica (não houve a possibilidade de realizar um estudo de incidência prévio devido à ausência de parametrização da totalidade dos focos de Enfermagem do âmbito da Saúde Mental e Psiquiátrica no sistema de informação em uso), foram criados programas de intervenção psicoterapêutica grupal para os focos de Enfermagem "Ansiedade", "Autoestima", e "Sono".

Para a avaliação dos programas de intervenção selecionaram-se participantes com recurso a técnicas de amostragem não probabilísticas por seleção racional, sendo que os utentes a excluir do programa de intervenção psicoterapêutica grupal deveriam ser aqueles que apresentassem um ou mais dos seguintes critérios de exclusão: não aceitação de participação na intervenção; défice cognitivo grave; elevada dificuldade de concentração; estado confusional; sintomatologia positiva exacerbada; agitação psicomotora; agressividade latente; hostilidade (Cordioli in Cordioli, 2008). O enfermeiro responsável pela seleção dos utentes era igualmente responsável por definir qual o foco de Enfermagem que deveria ser alvo de intervenção em cada semana, mediante a análise realizada pelo mesmo das necessidades de cuidados apresentadas pelos utentes.
Após a aplicação destes critérios, e ao longo do período de intervenção compreendido entre 23 de março de 2015 e 31 de dezembro de 2015, atingiu-se uma amostra total de 153 participantes.

\section{Considerações Éticas}

Todos os utentes que participaram nos programas de intervenção psicoterapêutica grupal foram respeitados na sua autonomia, tendo aceitado participar nos mesmos sabendo que da sua recusa não resultariam quaisquer repercussões para o seu tratamento. Os dados recolhidos foram documentados nos processos clínicos dos utentes (na medida em que as intervenções em causa se inserem no domínio da prestação de cuidados habitualmente disponibilizada aos utentes) mas, para efeitos de análise estatística e divulgação dos resultados, todos os dados foram codificados de modo a garantir a sua confidencialidade.

\section{Análise de Dados}

Para a caraterização da amostra recorreu-se à análise de frequências absolutas e relativas. Para a avaliação dos eventuais ganhos em saúde (comparação dos resultados - scores - antes e após a realização dos programas de intervenção psicoterapêutica) recorreu-se à estatística inferencial, particularmente à utilização de testes $t$ de Student para amostras emparelhadas e à análise do effect size ( $\mathrm{d}$ de Cohen). O nível de significância estatística, no presente trabalho, foi previamente fixado em 0,05. A análise dos dados foi realizada com recurso ao IBM SPSS Statistics 21.0 para Macintosh (IBM Corp. Released 2012).

\section{RESULTADOS}

\section{Caraterização da Amostra}

As caraterísticas dos participantes no estudo foram alvo de análise a três níveis: número de participantes em função do foco de Enfermagem ao qual foi dirigida a intervenção (Quadro 2); número de sessões nas quais os utentes participaram ao longo do programa de intervenção (Quadro 3); distribuição da amostra por género (Quadro 4).

Quadro 2 - Número de Participantes em Função do Foco de Enfermagem

\begin{tabular}{|c|c|c|}
\hline Focos de Enfermagem & $\mathbf{n}$ & \% do Total da Amostra \\
\hline Autoestima & 44 & 28,76 \\
\hline Ansiedade & 37 & 24,18 \\
\hline Sono & 72 & 47,06 \\
\hline Total & 153 & 100 \\
\hline
\end{tabular}


Quadro 3 - Número de Sessões nas quais os Utentes Participaram

\begin{tabular}{|c|c|c|}
\hline $\begin{array}{c}\text { Número de Sessões por } \\
\text { Utente }\end{array}$ & $\mathbf{n}$ & \% do Total da Amostra \\
\hline 3 Sessões & 1 & 0,65 \\
\hline 4 Sessões & 56 & 36,60 \\
\hline 5 Sessões & 96 & 62,75 \\
\hline Total & 153 & 100 \\
\hline
\end{tabular}

Quadro 4 - Distribuição da Amostra por Género

\begin{tabular}{|c|c|c|}
\hline Género & n & \% do Total da Amostra \\
\hline Feminino & 84 & 54,90 \\
\hline Masculino & 69 & 45,10 \\
\hline Total & 153 & 100 \\
\hline
\end{tabular}

\section{Ganhos em Saúde}

No que concerne ao programa de intervenção psicoterapêutica grupal dirigido ao foco de Enfermagem "Autoestima", não se verificaram diferenças estatisticamente significativas entre os scores inicial e final da Escala de Autoestima de Rosenberg ( $t=1,78$; $g l=43$; $\mathrm{p}=0,083)$. Igualmente, o nível de significância prática foi reduzido $(\mathrm{d}=0,25)$.

Relativamente ao programa de intervenção psicoterapêutica grupal dirigido ao foco de Enfermagem "Ansiedade", verificaram-se diferenças estatisticamente significativas entre os scores inicial e final da Escala de Avaliação da Ansiedade de Hamilton ( $\mathrm{t}=6,28 ; \mathrm{gl}=36$; $\mathrm{p}<0,001)$. O nível de significância prática foi elevado $(\mathrm{d}=0,87)$.

Finalmente, em relação ao programa de intervenção psicoterapêutica grupal dirigido ao foco de Enfermagem "Sono", verificaram-se diferenças estatisticamente significativas entre os scores inicial e final do Índice de Qualidade do Sono de Pittsburgh ( $\mathrm{t}=4,46 ; \mathrm{gl}=71$; $\mathrm{p}<0,001)$. Adicionalmente, foi identificado um nível elevado de significância prática $(\mathrm{d}=0,85)$.

\section{DISCUSSÃO}

Analisando os resultados alcançados, é possível verificar que os programas de intervenção psicoterapêutica grupal parecem ter sido, de forma global, eficazes. Assim, a única exceção foi verificada no programa de intervenção dirigido ao foco de Enfermagem "Autoestima”. Realizando uma análise ao programa de intervenção em causa e a quais podem ter sido os fatores que estiveram na origem do seu relativo insucesso, a abordagem ao foco (centrada na técnica de resolução de problemas e, portanto, tendo por base as dificuldades apresentadas pelos utentes) parece não ter sido eficaz na medida em que, de acordo com a literatura, a autoestima deve ser abordada, sob o ponto de vista da intervenção, de uma forma positiva (Mruk, 2013). Considerando estes dados, o programa de intervenção em causa foi alvo de alterações que, de acordo com uma análise preliminar aos resultados mais recentes, aponta já para uma melhoria significativa dos mesmos.

Relativamente aos restantes programas de intervenção psicoterapêutica grupal, dirigidos aos focos de Enfermagem "Ansiedade" e "Sono", os resultados são francamente positivos. Tal está em linha com outros estudos que avaliaram a eficácia de programas de intervenção psicoterapêutica grupal que visavam reduzir a ansiedade (e.g., van Ingen \& Novicki, 2009) e melhorar a qualidade do sono (e.g., Belber, De La Fuente, \& Canellas, 2013).

Considerando os resultados tendencialmente positivos que têm sido alcançados importa, no futuro, desenvolver programas de intervenção psicoterapêutica grupal que permitam dar resposta a outros focos de Enfermagem. Nesse sentido, em outubro de 2015 foi já criado um programa de intervenção psicoterapêutica grupal dirigido ao foco "Abuso de Álcool", que tem como objetivo reduzir o risco de recaída dos utentes internados para desabituação do consumo de álcool. Apesar de já existirem alguns resultados (positivos) relativos a esse programa, os mesmos não são apresentados no presente artigo na medida em que, até ao final de 2015, apenas três utentes tinham sido alvo de avaliação inicial e final e, como tal, os achados existentes carecem ainda de confirmação numa amostra de maior dimensão.

\section{Limitações}

O presente trabalho, por não se tratar primariamente de um trabalho de investigação, apresenta diversas limitações metodológicas, pelo que os resultados devem ser analisados com ponderação. Assim, desde logo, importa referir que os achados apresentados não podem ser atribuídos, única e exclusivamente, aos programas de intervenção psicoterapêutica grupal realizados, na medida em que existem variáveis estranhas não controladas que podem ter afetado os mesmos (como, por exemplo, as alterações realizadas na terapêutica medicamentosa ao longo do período dos programas de intervenção). 
Assim, seria importante a realização de um estudo clínico controlado randomizado na medida em que o recurso a uma técnica de amostragem probabilística, o controlo de algumas variáveis, e a existência de um grupo de controlo permitiriam a obtenção de resultados com nível de evidência significativamente superior.

\section{CONCLUSÕES}

O presente trabalho aponta para a tendencial existência de benefícios para os utentes de um Serviço de Internamento de Agudos de Psiquiatria da realização de programas de intervenção psicoterapêutica grupal dinamizados por enfermeiros especialistas em Enfermagem de Saúde Mental. Nesse sentido, e pese embora as limitações apresentadas anteriormente, importa utilizar os achados apresentados como forma de argumentar junto dos gestores hospitalares e dos decisores políticos acerca do potencial e capacidade destes mesmos enfermeiros especialistas para executar intervenções deste género, tendo como fim último a melhoria dos cuidados de saúde disponibilizados aos cidadãos. A concretização destes programas de intervenção apenas foi possível devido à existência de um enfermeiro especialista em Saúde Mental no contexto da prática clínica onde o presente trabalho foi realizado que, durante os dias úteis, tem como plano de trabalho único para o turno da manhã a consecução dos mesmos. Assim, este parece ser também um trabalho que pode ser utilizado para argumentar relativamente à importância das dotações seguras nos contextos de Saúde Mental e Psiquiatria, como forma de otimizar a prestação de cuidados aos utentes, permitindo que estes tenham acesso a cuidados especializados.

No futuro, seria importante tentar perceber os efeitos deste tipo de programas de intervenção nas taxas de reinternamento de utentes em contextos de Saúde Mental e Psiquiatria. Desse modo, e caso se verificassem resultados positivos, poder-se-iam somar os benefícios económicos aos evidentes benefícios sociais deste tipo de intervenção.

\section{IMPLICAÇÕES PARA A PRÁTICA CLÍNICA}

O presente trabalho pode ser considerado relevante para a prática clínica na medida em que a apresentação de resultados positivos relativos à consecução de programas de intervenção psicoterapêutica grupal dinamizados por enfermeiros especialistas em Enfermagem de Saúde Mental pode ser considerada como uma base para que este tipo de práticas se tornem mais comuns e possam vir a ser adotadas noutros contextos da prática clínica de Saúde Mental e Psiquiatria. Para além disso, a disseminação de boas práticas de Enfermagem pode ser assumida como uma mais-valia, na medida em que parece existir um desfasamento substancial entre as boas práticas realizadas em contextos da prática clínica e a divulgação das mesmas, tornando pouco visíveis as intervenções autónomas de Enfermagem e os ganhos em saúde que das mesmas podem advir. Finalmente, o trabalho desenvolvido pode ser considerado relevante para a gestão em Enfermagem na medida em que este vem reforçar a ideia de que com dotações mais adequadas é possível exercer a profissão com maior autonomia, prestando cuidados mais diferenciados e, dessa forma, contribuir para que a Enfermagem possa ser mais significativa para as pessoas.

\section{REFERÊNCIAS BIBLIOGRÁFICAS}

Belber, M., De La Fuente, L., \& Canellas, F. (2013). Insomnia group therapy. Sleep Medicine, 14(Suppl. 1), e87. doi: 10.1016/j.sleep.2013.11.181

Bulechek, G. M., Butcher, H. K., \& Dochterman, J. M. (2010). NIC: Classificação das Intervenções de Enfermagem ( $5^{\mathrm{a}}$ ed.). Loures: Lusodidacta.

Cordioli, A. V. (2008). As principais psicoterapias: Fundamentos teóricos, técnicas, indicações e contraindicações. In A. V. Cordioli (Coord.), Psicoterapias: Abordagens atuais. Porto Alegre, RS: Artmed.

Frank, J. D. (2006). What is psychotherapy? In S. Bloch (Ed.), An introduction to the psychotherapies (pp. 5976). Oxford, NY: Oxford University Press.

Frank, J. D., \& Frank, J. B. (1993). Persuasion and healing: A comparative study of psychotherapy ( $3^{a} \mathrm{ed}$.). Baltimore, MD: The John Hopkins University Press.

IBM Corp. Released. (2012) IBM SPSS Statistics for Macintosh, Version 21.0. Armonk, NY: IBM Corp.

Mruk, C. J. (2013). Self-esteem and positive psychology: Research, theory, and practice ( $4^{\mathrm{a}} \mathrm{ed}$.). New York, NY: Springer Publishing Company. 
